and colitis patients across $>100$ participating hospitals with a long-standing goal of fostering IBD Research to expedite clinical translation. For each patient recruited, clinical and selfreported phenotype data are collected, alongside plasma, serum and DNA samples for genetic analyses. The resulting data-rich panel is open to any investigators wishing to access data/samples or recall genotype-selected participants to donate further samples or trial novel therapies.

Methods Building the IBD BioResource panel: Patients' clinical data are collected at recruitment by the IBD team through a clinical data sheet based on the Montreal classification while health and lifestyle information are obtained via a patient questionnaire. All include demographics, smoking habits, general and IBD specific health questions, family history of IBD and non-IBD conditions, medication, treatment, surgery history and co-morbidities. The IBD BioResource panel also holds Whole Genome/Exome sequencing and GWAS data derived from its detailed biological samplings.

Accessing the IBD BioResource panel: The built-in panel of patients and their data is open to any investigators from science or industry. Following feasibility checks, submitted research applications are reviewed by the NIHR BioResource Scientific Advisory Board (SAB) based on scientific merits and projected benefit to patients. Upon study approval, patients meeting inclusion criteria are contacted by the NIHR BioResource team with a letter of invitation and a patient information leaflet. For volunteers willing to participate, appropriate arrangements are then made to gather information, collect fresh biological samples or engage patients in intervention studies.

Results To date 26 'Stage 2 studies' applied to utilise the IBD BioResource, 18 of which are currently active, 4 completed and 4 pending approval. Of the 26 applications, $\sim 20 \%$ requested access to anonymised samples/data while the remaining $\sim 80 \%$ required involvement of participants. Applications are national, with 7 from London, 6 from Cambridge, 4 from Oxford, 2 from Leeds. 2 from Pharma and 1 from Manchester, Exeter, Wolverhampton, Liverpool and Edinburgh. Field of studies include genetics, predictors and management of IBD; environmental, microbial and immunological contributors, fertility and well-being.

Conclusion The IBD BioResource and its network are on course to facilitating IBD Research through access to their panel and furthering knowledge/understanding of IBD for the benefit of Crohn's and colitis patients.

\section{P135 REAL-WORLD EFFECTIVENESS AND SAFETY OF USTEKINUMAB FOR THE TREATMENT OF CROHN'S: THE SCOTTISH USTEKINUMAB COHORT}

\footnotetext{
${ }^{1}$ Nikolas Plevris*, ${ }^{2}$ Andrew Robertson, ${ }^{1}$ James Fulforth, ${ }^{3}$ Rebecca Hall, ${ }^{1,4}$ Philip Jenkinson, ${ }^{5}$ Iona Campbell, ${ }^{6}$ Claire Kane, ${ }^{1}$ Ian Arnott, ${ }^{2}$ Diarmid Sutherland, ${ }^{7}$ Jennifer Veryan, ${ }^{2}$ Jennifer Saunders, ${ }^{8}$ Wai Lam, ${ }^{5}$ Graham Naismith, ${ }^{1}$ Cher Chuah, ${ }^{9}$ Christopher Kelly, ${ }^{9}$ David Watts, ${ }^{7}$ Daniel Gaya, ${ }^{8}$ Jonathan Macdonald, ${ }^{8}$ John-Paul Sennan, ${ }^{10}$ Hasnain Jafferbhoy, ${ }^{3}$ Craig Mowat, ${ }^{6}$ Gillian Bain, ${ }^{1}$ Gareth Jones, ${ }^{1}$ Charlie Lees. ${ }^{1}$ Edinburgh IBD Unit, Edinburgh, UK; ${ }^{2}$ Department of Gastroenterology, University Hospital Hairmyres, East Kilbride, UK; ${ }^{3}$ Department of Gastroenterology, Ninewells Hospital, Dundee, UK; ${ }^{4}$ Department of Gastroenterology, Raigmore Hospital, Inverness, UK; ${ }^{5}$ Department of Gastroenterology, Royal Alexandra Hospital, Paisley, UK; ${ }^{6}$ Department of Gastroenterology, Aberdeen Royal Infirmary, Aberdeen, UK; ' Department of Gastroenterology, Glasgow Royal Infirmary, Glasgow, UK; ${ }^{8}$ Department of Gastroenterology, Queen Elizabeth Hospital, Glasgow, UK; ${ }^{9}$ Department of Gastroenterology, Forth Valley Hospital, Larbert, UK; ${ }^{10}$ Department of Gastroenterology, Victoria Hospital Kirkcaldy, Kirkcaldy, Flfe, UK
}

Introduction Ustekinumab (UST) is an anti-IL12/23 biologic licensed for the treatment of moderate to severe Crohn's disease (CD). The aims of this study were to establish the longterm real-world effectiveness and safety of UST for the treatment of CD in a large UK cohort.

Methods This was a multicentre retrospective cohort study including 8 NHS health-boards in Scotland. Patients treated with UST between Nov 2015 and Jun 2019 were identified. Inclusion criteria included: a diagnosis of CD; active symptoms attributed to $\mathrm{CD}$ with objective evidence of mucosal inflammation (CRP $>5 \mathrm{mg} / \mathrm{L}$ or faecal calprotectin $\geq 250$ $\mu \mathrm{g} / \mathrm{g}$ or inflammation on endoscopy/MRI); completion of standard induction; week 8 review \pm further follow up. Clinical assessments were performed based on physician global assessment (response was defined as $\geq 50 \%$ reduction in CD-related symptoms and remission defined as complete resolution of all CD-related symptoms). Mucosal healing was defined as absence of ulceration/erosions on ileo-colonoscopy or no inflammation on MRI if ileo-colonoscopy was not possible (eg. B2 disease). Deep remission was defined as clinical remission plus mucosal healing. Perianal response was determined by follow up MRI (reduction in enhancement, closure or fibrosis of tract compared to baseline MRI). Rates of serious adverse events (discontinuation of UST, hospitalisation or death) during follow up were described quantitively.

Results A total of 216 patients $(57.9 \%$ female; median age 39.0 years, IQR 28.8-51.8; median disease duration 9.9 years, IQR 6.0-16.5) with a median follow up of 35.0 weeks (IQR 17.4-52.0) were included. The majority of patients had ileocolonic disease (L1, 19.9\%; L2, 23.1\%; L3, 56.9\%) and an inflammatory phenotype (B1, 43.1\%; B2, 41.2\%; B3, 15.7\%). A total of $98.6 \%$ of patients had previously been exposed to a biologic and 55.1\% had undergone previous surgery. Seventy-one percent of patients received 8 -weekly maintenance dosing, whilst $25.5 \%$ and $40.7 \%$ of patients were also receiving an immunomodulator and/or steroids at initiation, respectively. At week 8, clinical response and remission rates were $45.4 \%$ and $6.0 \%$, respectively. Twelve-month cumulative rates of clinical remission, mucosal healing $(n=123)$ and deep remission $(n=123)$ were $32.0 \%, 32.7 \%$ and $19.3 \%$, respectively (figure 1 ). In patients with active perianal disease at initiation $(n=37)$, 12-month cumulative rates of perianal response were $53.1 \%$. During 140 patient years of follow up
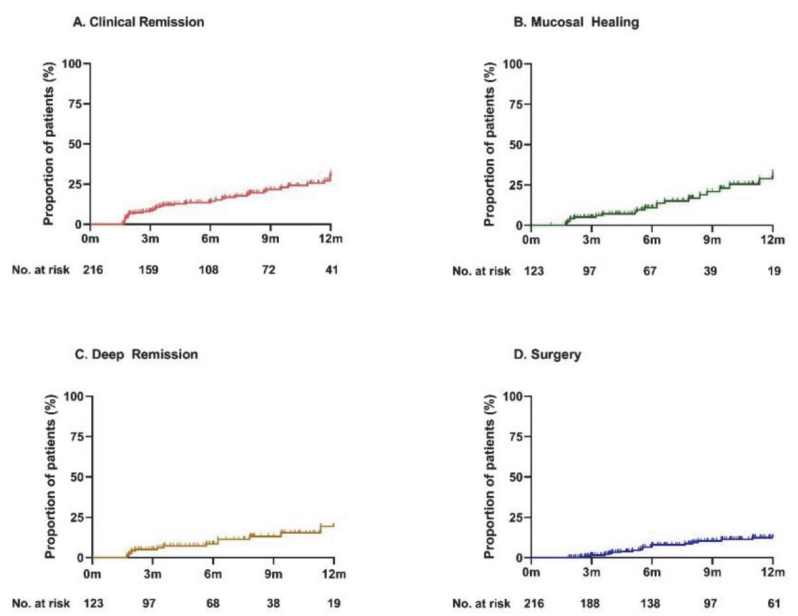

Abstract P135 Figure 1 
(PYF), 19 patients experienced a serious adverse event (13.6 per 100 PYF).

Conclusions We have shown in a large real-world cohort of complex, treatment refractory CD patients that UST is a safe and effective treatment option

\section{P136 IMMUNOMODULATORY MECHANISMS OF FMT IS ASSOCIATED WITH CLINICAL RESPONSE IN UC - RESULTS FROM STOP-COLITIS}

\begin{abstract}
${ }^{1}$ Mohammed Nabil Quraishi*, ${ }^{2}{ }_{\text {Ye }} 00,{ }^{3}$ Andrew D Beggs, ${ }^{2}$ David Withers, ${ }^{3}$ Animesh Acharjee, ${ }^{1}$ Naveen Sharma, ${ }^{1}$ Susan Manzoor, ${ }^{4}$ Nicholas J Loman, ${ }^{5}$ Konstantinos Gerasimidis, ${ }^{6}$ Richard Hansen, ${ }^{3}$ Georgios Gkoutous, ${ }^{7}$ Ailsa L Hart, ${ }^{8}$ Daniel R Gaya, 'Tariq H Iqbal. 'University of Birmingham Microbiome Treatment Centre, Birmingham, UK; ${ }^{2}$ Institute of Immunology and Immunotherapy, University of Birmingham, UK, UK; ${ }^{3}$ Institute of Cancer and Genomic Sciences, University of Birmingham, UK; ${ }^{4}$ Institute of Microbiology and Infection, University of Birmingham, UK; ${ }^{5}$ Human Nutrition, University of Glasgow, Glasgow, UK; ${ }^{6}$ Department of Paediatric Gastroenterology, Royal Hospital for Children Glasgow, Glasgow, UK; ${ }^{7} I B D$ Unit, St Marks Hospital, London, UK; ${ }^{8}$ Gastroenterology Unit, Glasgow Royal Infirmary, Glasgow, UK
\end{abstract}

\subsection{6/gutjnl-2020-bsgcampus.211}

Background Studies of faecal microbiota transplantation (FMT) for treating ulcerative (UC) have shown promising results, however mechanisms by which FMT modulates inflammation remain unexplored. Through an open-label pilot of FMT in UC (STOP-Colitis) we conducted a sub-study to explore changes in host colonic mucosal immune cell subsets and gene expression following FMT.

Methods Patients in this study received 8 infusions of FMT over an 8 week period. Colon biopsies were obtained at baseline and at end of the study. Immunophenotyping of colonic lamina propria mononuclear cells (LPMC) and RNA sequencing was conducted on colon biopsies for differential gene expression analysis.

Results 17 patients were recruited to this sub-study of which 12 completed study per protocol. Response (reduction in MAYO score) was seen in $67 \%(8 / 12)$ of patients. Analysis of colonic LPMC populations revealed a significant increase in regulatory $\mathrm{T}$ cells (Tregs, CD4+CD25+CD127lowFoxP3+; $\Delta 5.02 \%$; $\mathrm{p}<0.01)$, effector Tregs (CD4+CD25+CD127CCR7-CD45RA-; $\Delta 12 \% ; \mathrm{p}<0.001$ ), gut homing Tregs (CD4 $+\mathrm{CD} 25+\mathrm{CD} 127-\mathrm{CCR} 7-\mathrm{CD} 45 \mathrm{RA}-\alpha 4+; \quad \Delta 18.55 \% ; \quad \mathrm{p}<0.01)$ and IL-10 producing CD4 cells $(\Delta 2.16 \% ; \mathrm{p}=0.04)$ in responders following FMT. There was a significant reduction in mucosal Th17 (CD4+CD161+CCR6+; $\Delta-7.61 \% ; \mathrm{p}=0.017$ ), IL-17 producing CD4 $(\Delta 7.69 \% ; p=0.05)$ and CD8 $(\Delta-5.18 \%$; $\mathrm{p}=0.04)$ populations in FMT responders. Colonic mucosal gene expression and pathway analysis demonstrated that response to FMT was associated with significant downregulation of host antimicrobial defence response mainly REG and defensin family of anti-microbial peptides, pathogen-associated molecular pattern receptors, MHC class II antigen presentation genes and proinflammatory pathways. There was significant upregulation of butanoate and propionate metabolic pathways in FMT responders.

Conclusion Response to FMT is associated with a significant increase in mucosal gut homing Tregs and butanoate metabolism along with a reduction in Th17 cells and multiple antimicrobial defence and proinflammatory pathways. Exploring microbial mediators in FMT which influence immunometabolism are now under investigation to underpin novel biotherapeutic approaches.

\section{P137 THE IMPACT OF IBD FATIGUE ON HEALTH-RELATED QUALITY OF LIFE: A QUALITATIVE SEMI-STRUCTURED INTERVIEW STUDY}

${ }^{1}$ Shellie Radford*, ${ }^{2}$ Wladyslawa Czuber-Dochan, ${ }^{1}$ Gordon W Moran. ${ }^{1}$ NIHR Nottingham Biomedical Research Centre and University Of Nottingham, Nottingham, UK; ${ }^{2}$ Kings College London, Florence Nightingale Faculty of Nursing, midwifery and palliative care, London, UK

\subsection{6/gutjnl-2020-bsgcampus.212}

Background Fatigue is a frequently reported symptom of inflammatory bowel disease (IBD), experienced by patients with active disease and in remission. Fatigue related to chronic conditions plays a significant negative role in Health-Related Quality of Life (HRQoL), but patients' experience of this have not been described in IBD. We aimed to explore experience of IBD fatigue and its impact on HRQoL in adults diagnosed with IBD.

Methods Qualitative, semi-structured in-depth interviews were conducted with adults with IBD in remission, recruited from out-patient clinics in the United Kingdom. Eligibility and medical history were confirmed at recruitment. Interviews were audio-recorded and transcribed verbatim. Thematic analysis was employed to analyse the data using NVivo 12 software.

Results Fourteen participants (eight females, average age 37.3 years old, range 21-64) were interviewed. All identified themselves as a 'White British', average length of living with IBD fatigue was 10.9 years (range 9 months -17 years). Twelve participants reported that fatigue was constant and two reported intermittent fatigue. There were three key themes that reflect the patient experience: 1) 'The new normal' established through attempts to adapt daily life and acceptance of IBD fatigue impact on daily life and therefore impact on HRQoL. HRQoL is negatively impacted by lack of feelings of fulfilment, not being able to continue on as before the onset of IBD fatigue and a negative perception of self in comparison to others without IBD fatigue; 2) 'Energy as a resource' describes participants attempts to better manage fatigue on a daily basis through planning and prioritising tasks, often prioritising employment or education above social or leisure activities; 3) 'Keeping healthy' encompasses participants beliefs that good nutrition, good general health and keeping active allow them to generate energy more easily allowing some situational control where they have little control over IBD symptoms and subsequently improve HRQoL. Participants reported a mix of physical activities that improved HRQoL, however none reported a specific programme of exercise.

Conclusion Adults with IBD fatigue try to establish a sense of 'new' normality, through maintaining the same or similar, level of activities related to employment or education. However, this is often at the expense of personal, social and leisure activities. The study also indicates that perceptions of conservation of energy through planning and prioritising tasks and high levels of social support were associated with better selfreported HRQoL. Further research is required to explore physical activity-based intervention in relation to IBD fatigue, with use of validated fatigue and HRQoL measures. 